Research Paper

\title{
Risk Factors for Subclinical Atherosclerosis in Diabetic and Obese Children
}

\author{
Maria Felicia Faienza ${ }^{\natural}$, Angelo Acquafredda ${ }^{1}$, Riccardina Tesse ${ }^{1}$, Vincenza Luce ${ }^{1}$, Annamaria Ventura ${ }^{1}$, \\ Nicola Maggialetti², Mariantonietta Monteduro², Paola Giordanoㄴ, Luciano Cavallo ${ }^{1}$ \\ 1. Department of Biomedical Sciences and Human Oncology, University of Bari "A. Moro", Bari, Italy; \\ 2. Department of Diagnostic Imaging, University of Bari “A. Moro", Bari, Italy.
}

$\triangle$ Corresponding author: Maria Felicia Faienza, M.D. Department of Biomedical Sciences and Human Oncology, University of Bari “A. Moro", Bari, Italy. Piazza G. Cesare 11, 70124, Bari, Italy. Phone: +39805593075 Fax: +39805592287 e-mail: mariafelicia.faienza@uniba.it.

() Ivyspring International Publisher. This is an open-access article distributed under the terms of the Creative Commons License (http://creativecommons.org/ licenses/by-nc-nd/3.0/). Reproduction is permitted for personal, noncommercial use, provided that the article is in whole, unmodified, and properly cited.

Received: 2012.09.07; Accepted: 2012.12.27; Published: 2013.02.14

\begin{abstract}
Background. Increased carotid intima-media thickness (cIMT) is considered a marker of early-onset atherosclerosis and it seems to predict cardiovascular events both in obese and diabetic subjects. We aimed to evaluate early signs of atherosclerosis and investigate for predisposing factors in children and adolescents affected by type I diabetes (TIDM) or obesity, comparing them with healthy controls.

Methods. Out of 7 I enrolled subjects (mean age I2.8 \pm 2.3 years), 26 had TIDM and 24 were obese, while 21 age- and sex-matched subjects acted as controls. cIMT was measured using standardized methods. Serum glucose, insulin, cholesterol, triglycerides and C-reactive protein levels were evaluated. An oral glucose tolerance test (OGTT) was performed in obese subjects.

Results. Diabetic and obese individuals showed higher cIMT mean values than healthy controls $(p<0.005)$. cIMT of the three examined segments correlated positively with fasting glucose levels and negatively with units of insulin $/ \mathrm{kg} /$ day administered in TIDM individuals. A positive correlation between insulin levels (basal and after oral glucose load) and cIMT of common, internal and external carotid artery was found in obese subjects $(p<0.03)$. High density cholesterol levels represented a protective factor for cIMT in this latter group of the study population.

Conclusions. Our findings show that cIMT correlates with high insulin levels (a sign of insulin resistance) in obese patients and with high fasting glucose levels (a sign of relative insulin deficiency) in TIDM subjects, confirming the need of reducing hyperinsulinism and monitoring blood glucose levels in these subjects to prevent atherosclerosis.
\end{abstract}

Key words: carotid intima-media thickness, atherosclerosis, type 1 diabetes, obesity, children.

\section{Introduction}

Type 1 diabetes (T1DM) and obesity are main risk factors for cardiovascular events [1, 2]. In particular, young adults with T1DM have an increased risk of early asymptomatic atherosclerosis and consequent cardiovascular morbidity and mortality [3-5]. Similarly, childhood obesity has been reported associated with biochemical and inflammatory factors that affect vascular endothelial function and that might confer a premature atherogenicity [6]. Moreover, the insulin resistance, key feature of obesity, metabolic syndrome and type 2 diabetes, results in an array of metabolic and vascular events which finally promote the development of atherosclerosis [7]. The atherosclerotic process starts in childhood and proceeds silently over a long period of time before clinical manifestations [4]. Carotid artery intima-media thickness (cIMT) is con- 
sidered a significant predictive marker of generalized atherosclerosis because of its correlation with coronary artery disease and it may predict future cardiovascular events in adults [4]. It is also recommended by the American Heart Association as a noninvasive imaging parameter for detecting atherosclerosis $[8,9]$. Previous studies reported an increase of cIMT in children with hypercholesterolemia as compared to controls [10]. However, data on this marker in obese children and in children and adolescents with T1DM have yielded conflicting results [11-18].

This study aimed to evaluate the presence of early signs of atherosclerosis and seek for predisposing factors in a group of T1DM children and adolescents treated with intensive insulin protocols and in a group of obese subjects.

\section{Patients and Methods}

We enrolled 71 subjects (mean age $12.86 \pm 2.38$ years) who attended our outpatients Unit of Pediatric Endocrinology. Out of them, 24 were obese (12 males, Body Mass Index -BMI for age and sex $>95^{\text {th }}$ centile), 26 subjects had T1DM (12 males, mean HbA1C $8.2 \%)$, with onset of the disease at the age of $2.9 \pm 0.2$ years, and 21 ( 11 males, BMI <85 th centile) were healthy controls recruited from relatives of our medical staff. All diabetic patients were on treatment with insulin injections 4 times/day, including three meal doses of insulin lispro and one dose of insulin glargine at bedtime. The subjects with clinical, biochemical and instrumental signs of heart disease or family history of hypertension or dyslipidemia were excluded.

A written informed consent was obtained from children's parents or their legal guardians. All the procedures used were in accordance with the guidelines of the Helsinki Declaration on Human Experimentation.

All patients underwent a general clinical examination, anthropometric measurements (height in $\mathrm{cm}$, weight in $\mathrm{kg}$, BMI expressed in $\mathrm{kg} / \mathrm{m}^{2}$ ) using Italian growth charts [19], and assessment of the pubertal and genital stage, according to Tanner criteria [20]. Both systolic (SBP) and diastolic blood pressure (DBP) were measured in all patients [21], while waist circumference was assessed only in obese subjects [22]. Blood glucose, insulin, total cholesterol (TC), high (HDL) and low (LDL) density lipoprotein cholesterol, triglycerides (TG) and C-reactive protein (CRP) were measured after overnight fasting in all subjects; fructosamine and $\mathrm{HbA1C}$ were assessed in diabetics. Values of TC, LDL, HDL and TG were considered in the normal range if within the $5^{\text {th }}$ and the $95^{\text {th }}$ percentile [23]. An oral glucose $(1.75 \mathrm{~g} / \mathrm{kg})$ tolerance test (OGTT) was performed in obese subjects recording basal levels of blood glucose and insulin and after 120 min.

B-mode high resolution ultrasonography (US) was performed with a PHILIPS HDI 5000 Sono CT US scanner, equipped with a linear transducer L 12-5, using a frequency of $18 \mathrm{MHz}$ suitable to study vascular walls. Participants were examined in the supine position with the head turning slightly to both sides. After identifying the bulb, longitudinal images of the common carotid artery and of the two traits that it generates (internal and external carotid) were obtained by combined B-mode imaging and color Doppler. The far wall was scanned and the resolution box was used to magnify this segment, $10-20 \mathrm{~mm}$ proximally to the carotid bulb, where were focused all measurements. Several images were acquired by using an anterior oblique angle ( $30^{\circ}$ from midline) and a lateral one $\left(100^{\circ}\right.$ from midline) [24]. The cIMT of the far wall was measured during end diastole (R-wave of the electrocardiogram). Three scans on both sides were selected, and nine $(3 \times 3)$ measurements of maximum far wall IMT were averaged; thus, the conclusive mean cIMT of each patient was calculated from a total of 18 measurements.

We defined the cIMT as thickened when $\geq 0.45$ $\mathrm{mm}$ in children younger than 10 years or if it was $\geq$ $0.55 \mathrm{~mm}$ in children older than 10 years and younger than 18 years [25-28].

\section{Statistical analysis}

Statistical analysis was performed using SPSS Software for Windows (version 15, Chicago, IL, USA). Data are expressed as means, standard deviations and percentages. Comparisons between groups or within the same group were made using the Kruskal-Wallis and the Wilcoxon-Mann-Whitney $U$ non parametric tests, respectively. Spearman rho correlation analysis and multiple regression models were used to include cardiovascular risk factors. A $p$ value $<0.05$ was considered statistically significant.

\section{Results}

Clinical features of the study population are shown in Table 1.

Age, height and stage of pubertal development did not differ significantly within the three studied groups, while, as expected, obese subjects had increased weight and BMI values compared to T1DM patients and controls. SBP and DBP were higher in obese and diabetics than in healthy subjects $(\mathrm{p}<0.015$ and $\mathrm{p}<0.002$, respectively). A positive correlation between SBP and BMI was found in obese individuals ( $r h o=0.26 ; \mathrm{p}<0.02)$ (data not shown). 
Table I. Clinical characteristics and anthropometric measurements of the study population.

\begin{tabular}{lllll}
\hline & T1DM (n=26) & OBESE $(\mathbf{n}=24)$ & CONTROLS (n= 21) & P value \\
& 9 prepubertal & 8 prepubertal & 8 prepubertal & $\mathrm{ns}$ \\
\hline Age (years) & $12.62 \pm 2.43$ & $13.07 \pm 2.04$ & $12.19 \pm 2.67$ & $\mathrm{~ns}$ \\
Gender Ratio & $0.8: 1$ & $1: 1$ & $1.1: 1$ & $<0.0001$ \\
Weight $(\mathrm{Kg})$ & $47.76 \pm 12.74$ & $74.28 \pm 24.47 \dagger$ & $48.22 \pm 8.68$ & $\mathrm{~ns}$ \\
Height $(\mathrm{cm})$ & $153.4 \pm 15.13$ & $152.54 \pm 3.01$ & $150.37 \pm 11.75$ & $<0.0001$ \\
BMI $\left(\mathrm{Kg} / \mathrm{m}^{2}\right)$ & $19.93 \pm 2.74$ & $31.02 \pm 4.96 \dagger$ & $21.18 \pm 2.33$ & $<0.015$ \\
SBP $(\mathrm{mmHg})$ & $113.65 \pm 2.28^{*}$ & $117.3 \pm 2.38^{*}$ & $107 \pm 2.54$ & $<0.002$ \\
DBP $(\mathrm{mmHg})$ & $73.26 \pm 10.29^{*}$ & $74.16 \pm 13.32^{*}$ & $64.04 \pm 3.39$ & $<0.0001$ \\
Blood Glucose $(\mathrm{mg} / \mathrm{dl})$ & $186.69 \pm 98.54^{\text {п }}$ & $80.03 \pm 10.71$ & $79.15 \pm 9.04$ & - \\
Insulinemia $0^{\prime}(\mu \mathrm{u} / \mathrm{ml})$ & $0.74 \pm 0,28$ & $32.2 \pm 14.2$ & - & - \\
Insulinemia $120^{\prime}(\mu \mathrm{u} / \mathrm{ml})$ & - & $141.2 \pm 56.54$ & - & - \\
Total cholesterol $(\mathrm{mg} / \mathrm{dl})$ & $157.25 \pm 27.95$ & $163.52 \pm 30.81$ & - & - \\
HDL cholesterol $(\mathrm{mg} / \mathrm{dl})$ & $50.17 \pm 11.14$ & $47.46 \pm 9.57$ & - & - \\
LDL cholesterol $(\mathrm{mg} / \mathrm{dl})$ & $90.14 \pm 19.47$ & $101.12 \pm 24.72$ & $57.24 \pm 19.16$ & - \\
Tryglicerides $(\mathrm{mg} / \mathrm{dl})$ & $54.00 \pm 21.20$ & $84.58 \pm 37.25 \dagger$ & - & $<0.001$ \\
HbA1C $(\%)$ & $7.99 \pm 1.71$ & - & $1 \pm 0.62$ & $<0.001$ \\
CRP $(\mathrm{mg} / \mathrm{dl})$ & $1.71 \pm 1.85$ & $3.64 \pm 2.60 \dagger$ & & \\
\hline
\end{tabular}

Parameters are shown as averages \pm standard deviation; BMI: body mass index; SPB: systolic blood pressure; DPB: diastolic blood pressure, HDL: high density level; LDL: low density level; CRP: C-reactive protein. * $p$ value $<0.05$ vs controls. $\dagger p$ value $<0.05$ vs T1DM and controls. np value $<0.05$ vs obese and controls.

Among T1DM patients, $77 \%$ of them had blood glucose concentration greater than $180 \mathrm{mg} / \mathrm{dl}, 54 \%$ had $\mathrm{HbA} 1 \mathrm{C}$ greater or equal to $8 \%$, and $73 \%$ had fructosamine levels greater than $300 \mu \mathrm{mol} / \mathrm{L}$; TC, HDL, LDL cholesterol, TG and CRP values were in the normal range. The levels of LDL cholesterol and TG were increased in $4.2 \%$ and $12.5 \%$ of the obese subjects, respectively. Their TG values were significantly higher compared to both diabetics and controls $(p<0.001)$. All obese patients had normal glucose tolerance with basal hyperinsulinemia (32.2 \pm 14.2 $\mu \mathrm{U} / \mathrm{ml})$ and after glucose loading $(141.2 \pm 56.54$ $\mu \mathrm{U} / \mathrm{ml})$.

cIMT values resulted higher in obese subjects than in diabetics $(p<0.05)$, and in both these groups compared to controls $(\mathrm{p}<0.007$ and $\mathrm{p}<0.02$, respectively) (Fig.1). Diabetic subjects showed a positive correlation between cIMT of the three studied segments and fasting blood glucose levels ( $r h o=0.47$, $\mathrm{p}<0.05)$. cIMT tended also to be negatively correlated with the units $/ \mathrm{kg} /$ day of insulin administered in the same group of subjects ( $r h o=-0.29, \mathrm{p}=0.05)$. The mean cIMT values were also significantly higher among diabetic boys than age-matched healthy control subjects $(0.51 \pm 0.05$ vs $0.49 \pm 0.05 ; p<0.02)$. No association between cIMT and age of diabetes onset was observed in this study.

We found a positive correlation between insulin levels (basal and after oral glucose load) and cIMT of common, internal and external carotid artery in the obese $(r h o=0.47, p<0.03)$. Furthermore, the obese boys had higher mean cIMT value than girls $(0.52 \pm 0.03$ vs $0.51 \pm 0.04 ; \mathrm{p}<0.05)$ and the mean cIMT was significantly higher among obese boys than age-matched healthy control subjects $(0.52 \pm 0.03$ vs $0.46 \pm 0.05$; $\mathrm{p}<0.01)$, but not among obese girls. cIMT values were also higher in subjects during puberty than at the prepubertal age (T1DM vs obese vs controls: $0.51 \pm$ 0.05 vs $0.52 \pm 0.04$ vs $0.46 \pm 0.05 ; p<0.005)$. The overall measurement of cIMT was also significantly different within studied groups stratifying the analysis by height: individuals less than $150 \mathrm{~cm}$ of height had higher values of c-IMT, compared to controls, than taller subjects $(0.53 \pm 0.03$ vs $0.52 \pm 0.04$ vs $0.47 \pm 0.05$; $\mathrm{p}<0.04$ ). Figure 2 shows the distribution of c-IMT values within the study groups in pubertal age males with height less than $150 \mathrm{~cm}(0.52 \pm 0.02$ vs $0.51 \pm 0.05$ vs $0.46 \pm 0.03 ; \mathrm{p}<0.02)$.

The multiple regression analysis performed in the obese group, selecting SBP, DBP, TC, HDL and TG as independent variables and cIMT as the dependent variable, showed that HDL cholesterol represented a protective factor for the thickening of cIMT (OR $=0.58$ with a $95 \%$ confidence interval of $0.31-0.73, \mathrm{p}=0.04$ ), while the other analyzed parameters did not seem to affect cIMT in this group of subjects. 


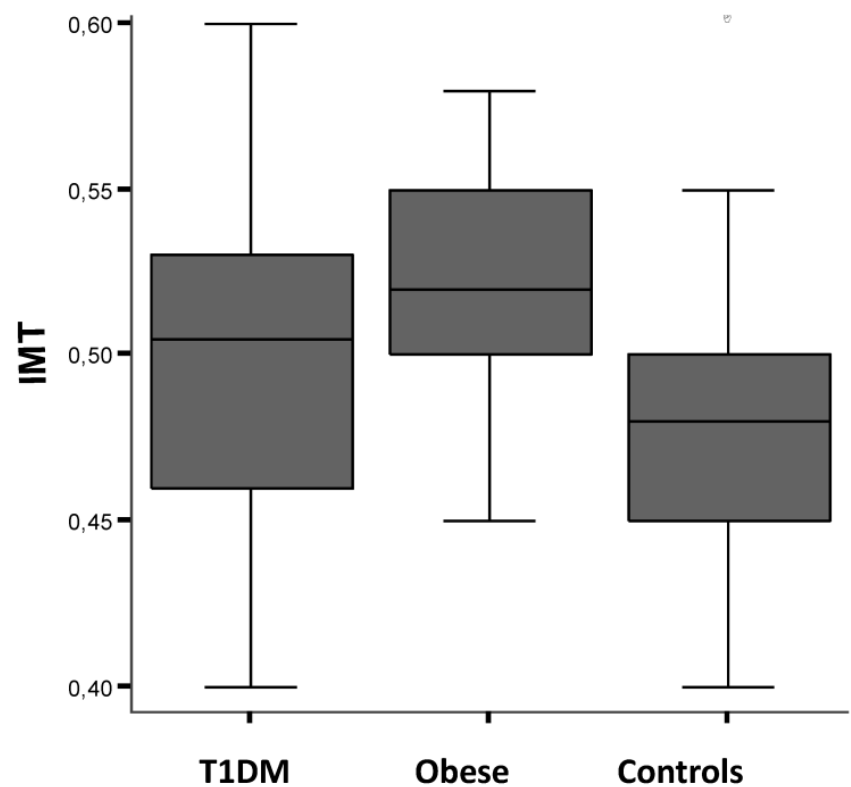

Figure I. Carotid artery intima-media thickness (cIMT) values by study population groups: type I diabetes mellitus (TIDM), obese subjects and healthy controls. See text for details.

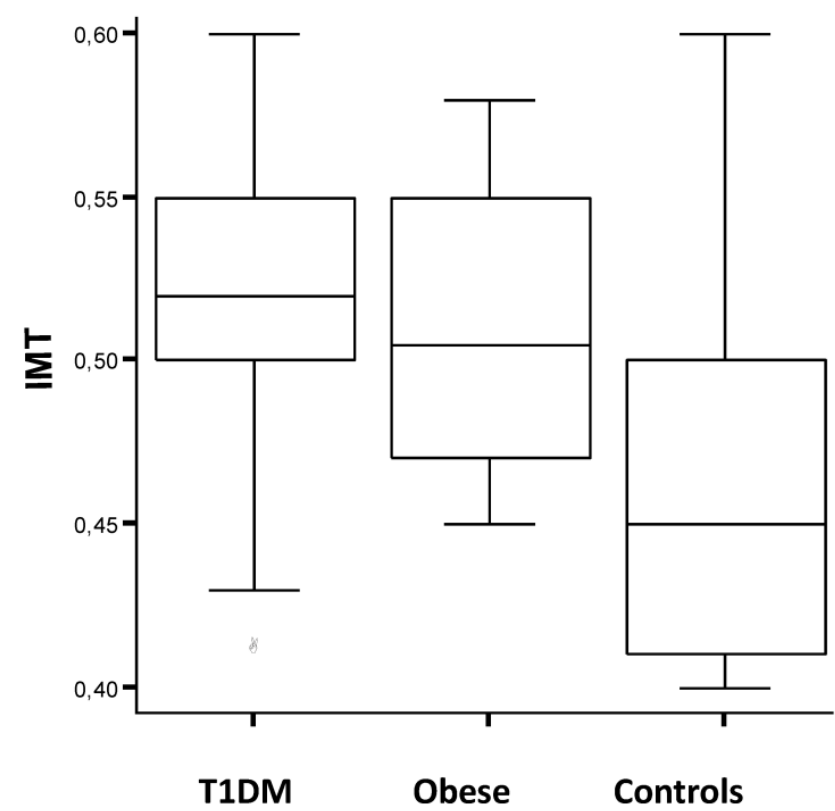

Figure 2. Carotid artery intima-media thickness (cIMT) values in pubertal age males of the study groups [Type I diabetes mellitus (TIDM), obese subjects and healthy controls] less than $150 \mathrm{~cm}$ tall. See text for details.

\section{Discussion}

In this study we identified subclinical atherosclerosis in T1DM patients and obese subjects as indicated by their significantly higher cIMT (mainly in boys) compared to that of healthy controls. Our re- sults suggest that the common pathogenetic factor involved in endothelial damage in obese and T1DM children and adolescents seems to be a reduced insulin function (insulin deficiency in diabetics and insulin resistance in obese patients, respectively). Insulin acts by modulating the release of vasodilator substances, such as nitric oxide and prostaglandins, from vascular endothelium, by both stimulating and inhibiting the sympathetic nervous system and by protecting smooth muscle cells in blood vessel from apoptosis induced by oxidative stress [29]. Thus the vasodilatory and antioxidant effects of insulin are depressed in case of insulin deficiency (i.e. type 1 diabetes) and insulin resistance (i.e. obesity) conditions.

It is well documented that cIMT correlates with coronary atherosclerosis [4], and that it represents an independent predictor of future cardiovascular events [9]. It also well correlates with parameters of invasive examination tools, such as coronary angiography and intravascular ultrasound $[4,30]$.

Studies on increased cIMT in children and adolescents affected with obesity, T1DM, dyslipidemia, hypertension and chronic renal failure, compared to healthy controls, have yielded conflicting results [31, 32]. The different conclusions may be explained by the non-homogenous populations studied and by the variety of ultrasound methods used. Although in our study obese individuals showed only slightly elevated mean cIMT compared to subjects with T1DM, a significant high number of diabetic and obese patients had cIMT above the normal range, indicating that these patients showed early signs of atherosclerosis development compared with healthy controls.

The degree and duration of hyperglycemia has been associated with macrovascular complications and increased cardiovascular risk in adults and preadolescent children with T1DM [5, 33-36]. In the present study no association between cIMT and HbA1C at the time of visit (a marker of glycemic control) was observed in diabetic subjects. However, the positive correlation between cIMT (especially of the left common carotid intima) and fasting blood glucose suggests that accumulation of glycation end products, as a consequence of not optimal insulin therapy, increases oxidative stress and the subsequent cell damage. This hypothesis is also confirmed by the negative correlation, even if not statistically significant, between the values of cIMT and the units of insulin daily administered. In obese individuals, the positive correlation between basal and after oral glucose load insulin levels and cIMT shows that insulin resistance causes an imbalance of vascular homeostasis mechanisms responsible for the endothelial damage and dysfunction $[29,37]$. 
Previous studies have shown that cIMT is negatively correlated with HDL cholesterol levels and positively related with LDL levels, confirming that dyslipidemia has a prominent role in the pathogenesis of endothelial dysfunction with progression to atherosclerosis $[6,38,39]$. In line with data reported in literature, HDL cholesterol values represent a protective factor for cIMT in our obese population. On the other hand, although increased cIMT has been documented in children with daytime hypertension, as well as in those with nocturnal hypertension and T1DM [25, 40], in our study population no association between conventional cardiovascular risk factors, such as blood pressure or LDL cholesterol and cIMT was found. This could be explained by the short duration of the disease at the time of examination, and the young age of the study population.

The gender-related difference in cIMT measurements observed in our study (higher prevalence of atherosclerosis and cardiovascular disease among males compared to females) is in line with data reported in literature on the general population of children and adolescents [33, 34]. Furthermore, our obese subjects showed a higher value of cIMT during puberty than that recorded in those at the prepubertal age. This could be explained by the change of insulin sensitivity for increased activity of the growth hormone axis (GH)-IGF1, which worsens the condition of insulin resistance during puberty. Furthermore, recently Skilton and colleagues have positively correlated cIMT with height in non-diabetic children aged 8 -years [41], while in this study we have observed that diabetic children with high risk for increased cIMT are likely males at pubertal age less than $150 \mathrm{~cm}$ tall (Figure 2). Additional study in selected groups of children should be carried-out to get more conclusive results.

In conclusion, we found that diabetic and obese children present (as adults) an endothelial damage, confirmed by increased cIMT values. Our results point out the importance of identifying children with the highest risk for cardiovascular heart disease using noninvasive studies, even if more studies on wider populations are warranted to confirm the findings. We also observed that there is a positive correlation between high levels of basal and after oral loading glucose insulin levels (a sign of insulin resistance) and increased cIMT in obese, as well as between high fasting blood glucose levels (a sign of relative insulin deficiency) and cIMT in T1DM patients. Thus it is necessary to normalize blood glucose values by optimizing insulin therapy in diabetic subjects, and to reduce hyperinsulinism by weight loss and physical activity in obese subjects, in order to prevent future cardiovascular risks.

\section{Competing Interests}

The authors have declared that no competing interest exists.

\section{References}

1. Margeirsdottir HD, Larsen JR, Brunborg C, et al. High prevalence of cardiovascular risk factors in children and adolescents with type 1 diabetes: a population-based study. Diabetologia. 2008; 51: 554-561.

2. Burke GL, Bertoni AG, Shea S, et al. The impact of obesity on cardiovascular disease risk factors and subclinical vascular disease: the Multi-Ethnic Study of Atherosclerosis. Arch Intern Med. 2008; 168 :928-935.

3. Orchard TJ, Costacou T, Kretowski A, et al. Type 1 diabetes and coronary artery disease. Diabetes Care. 2006; 29: 2528-2538.

4. Dahl-Jørgensen K, Larsen JR, Hanssen KF. Atherosclerosis in childhood and adolescent type 1 diabetes: early disease, early treatment? Diabetologia. 2005; 48: 1445-1453.

5. Larsen J, Brekke M, Sandvik L, et al. Silent coronary atheromatosis in type 1 diabetic patients and its relation to long-term glycemic control. Diabetes. 2002; 51: 2637-2641.

6. Aggoun Y. Obesity, metabolic syndrome, and cardiovascular disease. Pediatr Res. 2007; 61: 653-659.

7. Cubbon RM, Kahn MB, Wheatcroft SB. Effects of insulin resistance on endothelial progenitor cells and vascular repair. Clin Sci. 2009; 117: 173-190.

8. Lorenz MW, von Kegler S, Steinmetz H, et al. Carotid intima-media thickening indicates a higher vascular risk across a wide age range: prospective data from the Carotid Atherosclerosis Progression Study (CAPS). Stroke. 2006; 37: 87-92.

9. Greenland P, Abrams J, Aurigemma GP, et al. Prevention Conference V: Beyond secondary prevention: identifying the high-risk patient for primary prevention: noninvasive tests of atherosclerotic burden. Writing Group III. Circulation. 2000; 101: 16-22.

10. Tonstad S, Joakimsen O, Stensland-Bugge E, et al. Risk factors related to carotid intima-media thickness and plaque in children with familial hypercholesterolemia and control subjects. Arterioscler Thromb Vasc Biol. 1996; 16: 984-991.

11. Iannuzzi A, Licenziati MR, Acampora C, et al. Increased Carotid Intima-Media Thickness and Stiffness in obese children. Diabetes Care. 2004; 27: 2506-2508.

12. Schiel R, Beltschikow W, Radòn $\mathrm{S}$, et al. Increased carotid intima-media thickness and associations with cardiovascular risk factors in obese and overweight children and adolescents. Eur J Med Res. 2007; 12: 503-508.

13. Maher V, O'Dowd M, Carey M, et al. Association of central obesity with early carotid intima-media thickening is independent of that from other risk factors. Intern J Obes. 2009; 33: 136-143.

14. Jarvisalo MJ, Putto-Laurila A, Jartti L, et al. Carotid artery intima-media thickness in children with type 1 diabetes. Diabetes. 2002; 51: 493-498.

15. Yavuz T, Akcay A, Omeroglu RE, et al. Ultrasonic evaluation of early atherosclerosis in children and adolescents with type 1 diabetes mellitus. J Pediatr Endocrinol Metab. 2002; 15: 1131-1136.

16. Rabago Rodriguez R, Gomez-Diaz RA, Tanus HJ, et al. Carotid intima media thickness in pediatric type 1 diabetic patients. Diabetes Care. 2007; $30: 2599-2602$

17. Gunczler P, Lanes R, Lopez E, et al. Cardiac mass and function, carotid artery intima-media thickness and lipoprotein (a) levels in children and adolescents with type 1 diabetes mellitus of short duration. J Pediatr Endocrinol Metab. 2002; 15: 181-186.

18. Atabek ME, Kurtoglu S, Pirgon O, et al. Arterial wall thickening and stiffening in children and adolescents with type 1 diabetes. Diabetes Res Clin Pract. 2006; 74: 33-40.

19. Cacciari E, Milani S, Balsamo A, et al. Italian cross-sectional growth charts for height, weight and BMI (2 to 20yr). J Endocrinol Invest. 2006; 29: 581-593.

20. Tanner JM, Whitehouse RH. Clinical longitudinal standards from birth to maturity for height, weight, velocity and stages of puberty. Arch Dis Child. 1976; 151: 170-176

21. National High Blood Pressure Education Program Working Group on High Blood Pressure in Children and Adolescents. The Fourth Report on the Diagnosis, Evaluation, and Treatment of High Blood Pressure in Children and Adolescents. Pediatrics. 2005; 114: 555-576. 
22. McCarty HD. Body fat measurement in children as predictors for metabolic syndrome: focus on waist circumference. Proc Nutr Soc. 2006; 65: 385-392.

23. Yip PM, Chan MK, Nelken J, et al. Pediatric reference intervals for lipids and apolipoproteins on the VITROS 5,1 FS Chemistry System. Clin Biochem. 2006; 39: 978-983.

24. Adams H, Amarenco P, Desvarieux M, et al. Mannheim intima-media thickness consensus. Cerebrovasc Dis. 2004; 18: 346-349.

25. Yang XZ, Liu Y, Mi J, et al. Pre-clinical atherosclerosis evaluated by carotid artery intima-media thickness and the risk factors in children. Chin Med J. 2007; 120: 359-362.

26. Järvisalo MJ, Jartti L, Näntö-Salonen K, et al. Increased aortic intima-media thickness: a marker of preclinical atherosclerosis in high-risk children. Circulation. 2001; 104: 2943-2947.

27. Touboul PJ, Hennerici MG, Meairs S, et al. Mannheim carotid intima-media thickness consensus (2004-2006). An update on behalf of the Advisory Board of the 3rd and 4th Watching the Risk Symposium, 13th and 15th European Stroke Conferences, Mannheim, Germany, 2004, and Brussels, Belgium, 2006. Cerebrovasc Dis. 2007; 23: 75-80.

28. Fang J, Zhang JP, Luo CX, et al. Carotid Intima-media thickness in childhood and adolescent obesity relations to abdominal obesity, high triglyceride level and insulin resistance. Int J Med Sci. 2010; 7: 278-283.

29. Muniyappa R, Quon MJ. Insulin action and insulin resistance in vascular endothelium. Curr Opin Clin Nutr Metab Care. 2007; 10: 523-530.

30. Sharma K, Blaha MJ, Blumenthal RS, et al. Clinical and research applications of carotid intima-media thickness. Am J Cardiol. 2009; 103: 1316-1320.

31. Margeirsdottir HD, Stensaeth KH, Larsen JR, et al. Early signs of atherosclerosis in diabetic children on intensive insulin treatment: a population-based study. Diabetes Care. 2010; 33: 2043-2048.

32. Lamotte C, Iliescu C, Libersa C, et al. Increased intima-media thickness of the carotid artery in childhood: a systematic review of observational studies. Eur J Pediatr. 2011; 170: 719-729.

33. Nathan DM, Lachin J, Cleary P, et al. Intensive diabetes therapy and carotid intima-media thickness in type 1 diabetes mellitus. N Engl J Med. 2003; 348: 2294-2303.

34. Nathan DM, Cleary PA, Backlund JY, et al. Intensive diabetes treatment and cardiovascular disease in patients with type 1 diabetes. $\mathrm{N}$ Engl J Med. 2005; 353: 2643-2653.

35. Babar GS, Zidan H, Widlansky ME, et al. Impaired endothelial function in preadolescent children with type 1 diabetes. Diabetes Care. 2011; 34: 681-685.

36. Larsen JR, Brekke M, Bergengen L, et al. Mean HbA1c over 18 years predicts carotid intima-media thickness in women with type 1 diabetes. Diabetologia. 2005; 48: 776-779.

37. Cubbon RM, Kahn MB, Wheatcroft SB. Effects of insulin resistance on endothelial progenitor cells and vascular repair. Clin Sci. 2009; 117: 173-190.

38. McGill HC Jr, McMahan CA. Determinants of atherosclerosis in the young. Pathobiological Determinants of Atherosclerosis in Youth (PDAY) Research Group. Am J Cardiol. 1998; 82: 30-36.

39. McGill HC Jr, McMahan CA, Zieske AW, et al. Associations of coronary heart disease risk factors with the intermediate lesion of atherosclerosis in youth. The Pathobiological Determinants of Atherosclerosis in Youth (PDAY) Research Group. Arterioscler Thromb Vasc Biol. 2000; 20: 1998-2004.

40. Lee SH, Kim JH, Kang MJ, et al. Implications of nocturnal hypertension in children and adolescents with type 1 diabetes. Diabetes Care. 2011; 34: 2180-2185.

41. Skilton MR, Sullivan TR, Ayer JG, et al. Carotid extra-medial thickness in childhood: early life effects on the arterial adventitia. Atherosclerosis. 2012; 222: 478-482. 